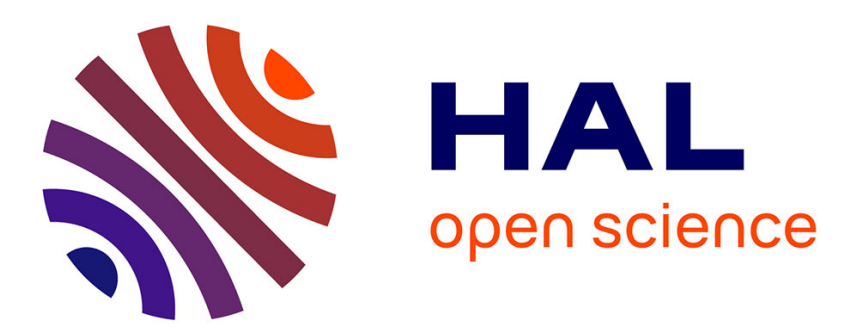

\title{
Unimolecular Fragmentation Properties of Thermometer Ions from Chemical Dynamics Simulations
}

\author{
Abdul Malik, Riccardo Spezia, William L Hase
}

\section{To cite this version:}

Abdul Malik, Riccardo Spezia, William L Hase. Unimolecular Fragmentation Properties of Thermometer Ions from Chemical Dynamics Simulations. Journal of The American Society for Mass Spectrometry, 2021, 32 (1), pp.169-179. 10.1021/jasms.0c00200 . hal-03109045

\section{HAL Id: hal-03109045 \\ https://hal.science/hal-03109045}

Submitted on 13 Jan 2021

HAL is a multi-disciplinary open access archive for the deposit and dissemination of scientific research documents, whether they are published or not. The documents may come from teaching and research institutions in France or abroad, or from public or private research centers.
L'archive ouverte pluridisciplinaire HAL, est destinée au dépôt et à la diffusion de documents scientifiques de niveau recherche, publiés ou non, émanant des établissements d'enseignement et de recherche français ou étrangers, des laboratoires publics ou privés. 


\title{
Unimolecular Fragmentation Properties of Thermometer Ions from Chemical Dynamics Simulations
}

\author{
Abdul Malik, ${ }^{a}$ Riccardo Spezia ${ }^{b, *}$ and William L. Hase ${ }^{a}$ \\ a Department of Chemistry and Biochemistry, Texas Tech University, Lubbock, Texas 79409- \\ 1061 USA. \\ ${ }^{\mathrm{b}}$ Laboratoire de Chimie Théorique, Sorbonne Université, UMR 7616 CNRS, 4 Place Jussieu, \\ 75005 Paris, France.
}

*correspondence to: riccardo.spezia@sorbonne-universite.fr

\begin{abstract}
Thermometer ions are widely used to calibrate the internal energy of the ions produced by electrospray ionization in mass spectrometry. Typically, benzylpyridinium ions with different substituents are used. More recently benzhydrylpyridinium ions were proposed for their lower bond dissociation energies. Direct dynamics simulations using M06-2X/6-31G(d), DFTB, and PM6-D3 are performed to characterize the activation energies of two representative systems: para-methyl-benzylpyridinium ion $\left(p-\mathrm{Me}-\mathrm{BnPy}^{+}\right)$and methyl,methyl-benzhydrylpyridinium ion $\left(\mathrm{Me}, \mathrm{Me}-\mathrm{BhPy}^{+}\right)$. Simulation results are used to calculate rate constants for the two systems. These rate constants and their uncertainties are used to find the Arrhenius activation energies and RRK fitted threshold energies which give reasonable agreement with calculated bond dissociation energies at the same level of theory. There is only one fragmentation mechanism observed for both systems, which involves $\mathrm{C}-\mathrm{N}$ bond dissociation via a loose transition state, to generate either benzylium or benzhydrylium ion and a neutral pyridine molecule. For $p$-Me$\mathrm{BnPy}^{+}$using DFTB and PM6-D3 the formation of tropylium ion, from rearrangement of benzylium ion, was observed but only at higher excitation energies and for longer simulation times. These observations suggest that there is no competition between reaction pathways that could affect the reliability of internal energy calibrations. Finally, we suggest using DFTB with modified-Arrhenius model in future studies.
\end{abstract}

Keywords: Thermometer ions; Unimolecular dissociation; Chemical Dynamics Simulations; Kinetic Theories; Benzylpyridinium Ions; Benzhydrylpyridinium Ions

\section{Introduction}

Understanding the characteristic temperature of ions produced by electrospray ionization (ESI) is of fundamental importance in mass spectrometry (MS). ${ }^{1}$ In fact, the fragmentation yield depends on the internal energy/temperature of such ions, but this quantity cannot be measured directly. To this end, the use of thermometer ions was introduced years ago: first studies by Cooks and co-workers focused on $\left(\mathrm{C}_{2} \mathrm{H}_{5}\right)_{4} \mathrm{Si}^{+}, \mathrm{Fe}(\mathrm{CO})_{5}{ }^{+}$, and $\mathrm{W}(\mathrm{CO})_{6}^{+},{ }^{2-4}$ while lately, benzylpyridinium (BnPy) ions were chosen as standard reference. ${ }^{5,6}$ The former ions are more suited for electron ionization or photoionization while the latter for ESI, which has become in recent years the most used ionization technique to study biomolecular systems. Due to the softness of the ionization in ESI, molecular structures are conserved, which is crucial to study peptides, sugars, proteins or other similar compounds. 
To obtain the internal energy distribution and thus the characteristic temperature of the thermometer ions, a crucial quantity to know is the threshold energy. Quantum chemistry calculations can provide the bond dissociation energy (BDE) as the energy difference between products and reactants. This is possible for the thermometer ions because they present oneproduct reactions characterized by a loose transition state (TS). Recently, a new set of thermometer ions was proposed, the benzhydrylpyridinium (BhPy) ions, which show lower activation energies than the original BnPy ions due to the addition of an extra phenyl ring that weakens the C-N bond by stabilizing the resultant benzhydrylium ion. ${ }^{7,8}$ In Scheme 1 we report the unimolecular fragmentations which characterize these two classes of thermometer ions.<smiles>[2H]c1ccc(C(c2ccc([2H])cc2)c2ccc([C@@H](C)c3ccc([2H])cc3)cc2)cc1</smiles>

Scheme 1. Fragmentation reactions for benzylpyridinium $\left(B n P y^{+}\right.$, upper panel) and benzhydrylpyridinium $\left(B h P y^{+}\right.$, lower panel) ions. In the present work, we have explicitly studied the methyl substituted ions $(R=M e)$.

Recently, chemical dynamics simulations were shown to be a useful tool to obtain unimolecular fragmentation energy thresholds. ${ }^{9,10}$ In particular, activation energies were obtained for fragmentation of relatively large peptides, for which it would be difficult to obtain these energies from potential energy surface scan. ${ }^{11,12}$ These values were obtained using Arrhenius phenomenological law and were found to be in agreement with barriers obtained from static calculations. RRK theory can also directly measure the threshold energies: a recent study on a model system has shown that the classical energy threshold differs from the quantum just by the (known) zero-point energy (ZPE) difference between the reactants and the products. ${ }^{13}$

The results of chemical dynamics rely on the method used to describe, on-the-fly, the potential energy surface (PES) of the system. Accurate theoretical methods like coupled cluster or even extended basis sets with density functional theory (DFT) or MP2 are not feasible for relatively large systems. To obtain enough statistical sampling, DFT (with small basis sets) or semiempirical Hamiltonians are often used in chemical dynamics simulations of unimolecular fragmentation. ${ }^{14,15}$ Semi-empirical Hamiltonians are particularly used to study large systems, since one needs an ensemble of trajectories and different energy points to obtain the desired quantities. These simulations are mainly aimed to model ion trap-like experiments (the socalled collisionally activated dissociation) by activating the system via an excess internal energy. Results are collected as a function of this energy, but it is hard to make a direct 
comparison with experimental energies. The use of thermometer ions can be a possible way to calibrate simulations as done in experiments.

To this end, we have first investigated the ability of chemical dynamics in reproducing the known fragmentation properties of two thermometer ions $\left(p-\mathrm{Me}-\mathrm{BnPy}^{+}\right.$and $\left.\mathrm{Me}, \mathrm{Me}-\mathrm{BhPy}{ }^{+}\right)$, belonging to the aforementioned BnPy and BhPy classes of compounds (they correspond to $\mathrm{R}=\mathrm{Me}$ in structures reported in Scheme 1). In particular, we have tested the semi-empirical Hamiltonians, tight-binding DFT (DFTB), and DFT with relatively small basis set.

Secondly, we studied how simple kinetic theories, like RRK and Arrhenius-like, are able to obtain threshold/activation energies and how they compare with static BDEs. The use of more sophisticated kinetic approaches (like, e.g. those developed by Troe ${ }^{16-19}$ and usually applied to small systems) will need the knowledge of molecular parameters which will be almost impossible for large systems, like peptides or sugars. This will be crucial to correctly calibrate simulations of new systems with respect to the thermometer ion. Finally, we can have direct access to the molecular fragmentation mechanisms and products and verify what is proposed experimentally.

Our work aims to study how the chemical dynamics approach simulates the fragmentation of two prototypical thermometer ions. In particular we focus on how simple kinetic theories can be applied in order to further use them to calibrate fragmentation simulations in energy. This will finally allow to be more quantitative when using simulations in modeling multiple-collision fragmentations of complex systems.

\section{Methods}

\subsection{Quantum Chemistry Calculations}

Geometries for $p$-Me-BnPy ${ }^{+}$and $\mathrm{Me}, \mathrm{Me}-\mathrm{BhPy}^{+}$were first optimized using Universal Forcefield ${ }^{20}$ followed by four semi-empirical methods, density functional based tight binding (DFTB) ${ }^{21}$ and density functional theory (DFT). Static calculations were performed for the two systems to compute the numerical frequencies and bond dissociation energies (BDE) with or without zero-point energies (ZPE). Semi-empirical methods include PM7, ${ }^{22}$ PM6-D3, ${ }^{23,24}$ $\mathrm{AM} 1,{ }^{25}$ and $\mathrm{RM} 1 .{ }^{26}$ DFTB with non-SCC ${ }^{27,28}$ (non-self-consistent charge) approximation was used. In particular we used the DFTB + implementation of $\mathrm{DFTB}^{29}$ containing many extensions to the original method. Slater-Koster parametrization set for C-H-N-O was used as reported by Gaus et al. ${ }^{30}$ For DFT, different GGA, meta-GGA, and LDA functionals were tested in preliminary calculations. $\mathrm{B} 3 \mathrm{LYP}^{31,32}$ and $\mathrm{M} 06-2 \mathrm{X}^{33}$ functionals were selected since they provided the best results in terms of BDEs. Thus, we considered for these two functionals different basis sets: 6-31G(d), 6-311+G(d,p) and cc-pVDZ. Chemical dynamics simulations were performed with 6-31G(d) (bigger basis sets are computationally too expensive to be practical). Note also that B3LYP and M06-2X are very popular functionals available in most quantum chemistry packages.

Semi-empirical calculations were performed with MOPAC $2016,{ }^{34}$ DFT calculations with NWChem $6.8,{ }^{35}$ and DFTB with DFTB+ software. ${ }^{36}$ 


\subsection{Chemical Dynamics Simulations}

The unimolecular dissociation dynamics of $p-\mathrm{Me}_{-} \mathrm{BnPy}^{+}$and $\mathrm{Me}, \mathrm{Me}-\mathrm{BhPy}{ }^{+}$were studied by chemical dynamics simulations, using PM6-D3, non-SCC DFTB and M06-2X/6-31G(d). These methods were chosen as the best representatives for semi-empirical, DFTB and DFT classes, based on the agreement with the experimental BDEs and the practicality of the computational expense.

Initial conditions were chosen as follows. First, the minimum energy geometries of the two systems were located for each different method. Then, the vibrational modes of these ions were randomly excited using classical microcanonical normal mode sampling, ${ }^{37}$ resulting in initial positions and velocities in internal coordinates which reflected the excess vibrational energy considered. In particular we used initial excitation energies between 10.84 and $23.85 \mathrm{eV}$. Ten excitation energies were considered in total: 10.84, 11.92, 13.00, 14.09, 15.18, 16.26, 17.34, 19.51, 21.68 and $23.85 \mathrm{eV}$. The rotational energy of the ions was sampled using a Boltzmann distribution at $300 \mathrm{~K}$. Then, initial positions and momenta were converted into Cartesian coordinates and trajectories were run with these initial conditions. Note that, for each method and system, this corresponded to an ensemble of initial conditions and thus an ensemble of resulting trajectories.

Trajectories were then produced by numerical integration of Newton equations of motion. In particular, we used the fourth-order symplectic algorithm ${ }^{38,39}$ with PM6-D3 Hamiltonian and velocity-Verlet ${ }^{40}$ algorithm with DFTB and M06-2X simulations. The time steps (listed in the Tables S1 and S2 in the Supporting Information), as well as the integration method, were chosen to achieve energy conservation within $2 \%$. Simulation times were set between 3 and 30 ps, as a function of the method and the energy. Details on simulation time length as well as the number of trajectories for each method, system and energy are listed in Tables S1 and S2. Note that these chemical dynamics simulations provide the time evolution of atomic positions (and momenta). The analysis of key atomic distances is used to identify the dissociation of a bond which represents a reactive trajectory. More details on simulation procedure are reported in a recent review article. ${ }^{9}$

PM6-D3 simulations were performed using the VENUS/MOPAC software package, in which MOPAC electronic structure computer program $^{34}$ is interfaced with VENUS chemical dynamics computer program. ${ }^{41,42}$ Simulations with DFTB + were done using in-house VENUS/DFTB + interface and M06-2X direct dynamics trajectories were performed with VENUS/NWChem interface.

\subsection{Kinetic Analysis}

From unimolecular fragmentation, it is possible to follow the abundance of the precursor ion as a function of time, by counting at each time step the number of non-reactive ions, $N(t)$. Since there was no re-crossing from products to reactants in the two systems, a trajectory was considered reactive once the $\mathrm{C}-\mathrm{N}$ bond distance reached 3.0 $\AA$. Monitoring this distance in time is crucial to follow the occurrence of the principal dissociation pathway of $p-\mathrm{Me}-\mathrm{BnPy}^{+}$and $\mathrm{Me}, \mathrm{Me}-\mathrm{BhPy}^{+}$.

The decay of the initial population can be fitted with an exponential function, 
$\frac{N(t)}{N(0)}=e^{-k t}$

obtaining the unimolecular rate constant, $k$ (where $N(0)$ is the number of trajectories and $t$ is the time). The fitted values of rate constants are reported with uncertainty providing $95 \%$ confidence interval.

The energy dependence can be reformulated in terms of temperature dependence, since for a classical system of $s$ harmonic oscillators the well-known relation holds: ${ }^{43}$

$E_{v}=s k_{B} T$

where $E_{v}$ is the vibrational energy, $k_{B}$ the Boltzmann constant and $T$ the temperature. Both systems, $p$-Me-BnPy ${ }^{+}$and $\mathrm{Me}, \mathrm{Me}-\mathrm{BhPy}^{+}$, have a large number of normal modes: 78 and 117, respectively. It has been established previously ${ }^{44}$ that for a molecule with a large number of normal modes $s$ such that $s \approx s-1$, the classical Rice-Ramsperger-Kassel-Marcus (RRKM) rate constant $k(E)$ becomes equivalent to the classical transition state theory (TST) rate constant $k(T)$ if $E_{o} / E<<1$, where $E_{o}$ is the unimolecular dissociation energy (in the Supporting Information we report the simple demonstration). ${ }^{45}$ Both of these criteria are met for the simulations reported here. Since there is only one dissociation pathway for both systems, its rate constant may be represented by the Arrhenius equation,

$k(T)=A e^{-\frac{E_{a}}{k_{B} T}}$

where $A$ is the pre-exponential factor (here temperature independent) and $E_{a}$, the activation energy, should correspond to the classical dissociation energy $E_{o}{ }^{46}$ and may be compared to the values given by the electronic structure theory methods used for the simulations. In previous studies, Arrhenius parameters have been derived from rate constants determined from direct and chemical dynamics simulations. ${ }^{11,12,44,47,48}$

This simple approach, the Arrhenius theory, disregards any temperature dependence of the activation energy and/or pre-exponential factor. Often, an improved version of the Arrhenius expression is used: ${ }^{49}$

$$
k(T)=A T^{m} e^{-D_{0} / k_{B} T}
$$

From Eq. 4 the activation energy (which is by definition $-k_{B}[d \ln k / d(1 / T)]$ ) is: $E_{a}=m k_{B} T+$ $D_{0}$ where $D_{0}$ is the dissociation energy and $T$ is the average temperature over the range of simulations. Note that when $E_{a}$ is lower than the barrier height of the underlying potential energy surface then $m<0$. It is well documented that activation energies obtained from Arrhenius plots often underestimate the true underlying barrier, in particular for loose TS. ${ }^{49}$ This improved expression is able sometimes to provide a better description of the reactivity, with a dissociation energy closer to the barrier height, since it introduces a temperature dependence in the pre-exponential factor. ${ }^{13,49,50}$ We have thus also used Eq. 4 to analyze simulation results.

Simulations are microcanonical, so they can be analyzed using the RRKM theory, and they are also Newtonians, so the classical RRK theory holds: 
$k(E)=\nu\left(\frac{E-E_{0}}{E}\right)^{s-1}$

where $v$ is a frequency factor (here an effective frequency) and $E_{o}$ the energy threshold. From equation 5 it is possible to obtain equation 3 as reported in the Supporting Information.

The three models (corresponding to equations 3-5) were considered and equations were fitted using rate constants and their standard deviations as obtained from simulations via the nonlinear least-squares Marquardt-Levenberg algorithm ${ }^{51,52}$ as implemented in the gnuplot software. ${ }^{53}$

\section{Results}

\subsection{Bond Dissociation Energy}

Fragmentation of thermometer ions is usually characterized by a specific bond dissociation energy (BDE) which depends on the substituent group and its location on the benzyl ring. ${ }^{6,54}$ For BnPy ions, the BDE is for the $\mathrm{C}-\mathrm{N}$ bond between the substituted benzylium cation and the neutral pyridine whereas for BhPy ions it is the dissociation energy between substituted benzhydrylium ion and the neutral pyridine (see Scheme 1, in the present case R=Me). ${ }^{55}$ It has been observed previously ${ }^{6,7,54}$ that this reaction proceeds through a loose TS, i.e. reverse activation barrier can be neglected and the BDE can be calculated as the zero-point energy difference between the reactants and the products.

We report in Table 1 the BDEs for both $p-\mathrm{Me}-\mathrm{BnPy}^{+}$and $\mathrm{Me}, \mathrm{Me}-\mathrm{BhPy}^{+}$as obtained from different theoretical methods and basis sets. Data are reported with and without ZPE to compare with the experimental as well as Newtonian simulation results. Note that the experimental values were obtained presuming that the reaction occurred via a loose TS. ${ }^{7,56}$

Table 1. Bond dissociation energies (in eV) for fragmentation of $p-\mathrm{Me}^{-\mathrm{BnPy}}{ }^{+}$and $\mathrm{Me}, \mathrm{Me}-$ $\mathrm{BhPy}^{+}$(values at $0 \mathrm{~K}$ ). In parentheses, we report values without $\mathrm{ZPE}$ correction.

\begin{tabular}{|c|c|c|}
\hline Method & $p^{-\mathrm{Me}-\mathrm{BnPy}}{ }^{+}$ & $\mathrm{Me}, \mathrm{Me}-\mathrm{BhPy}^{+}$ \\
\hline B3LYP/6-31G(d) & $1.92(2.08)$ & $0.88(1.00)$ \\
\hline B3LYP/6-311+G(d,p) & $1.80(1.96)$ & $0.78(0.92)$ \\
\hline B3LYP/cc-pVDZ & $1.89(2.06)$ & $0.42(0.61)$ \\
\hline M06-2X/6-31G(d) & $2.42(2.56)$ & $1.61(1.74)$ \\
\hline M06-2X/6-311+G(d,p) & $2.37(2.55)$ & $1.31(1.44)$ \\
\hline M06-2X/cc-pVDZ & $2.44(2.58)$ & $1.40(1.54)$ \\
\hline AM1 & $1.61(1.77)$ & $0.78(0.91)$ \\
\hline RM1 & $1.94(2.07)$ & $1.39(1.52)$ \\
\hline PM6-D3 & $2.09(2.21)$ & $1.41(1.53)$ \\
\hline PM7 & $1.93(2.08)$ & $1.26(1.38)$ \\
\hline DFTB & $2.11(2.31)$ & $1.45(1.60)$ \\
\hline DLPNO-CCSD $(\mathrm{T})^{\mathrm{a}}$ & $2.23(2.36)$ & $1.52(1.63)$ \\
\hline DLPNO-CCSD/CBS ${ }^{b}$ & 2.26 & 1.52 \\
\hline $\operatorname{CCSD}(\mathrm{T}) / 6-311++\mathrm{G}(\mathrm{d}, \mathrm{p})^{\mathrm{c}}$ & 2.267 & - \\
\hline Experiments & $2.26 \pm 0.13^{\mathrm{d}}$ & $1.55 \pm 0.13^{\mathrm{e}}$ \\
\hline
\end{tabular}


B3LYP/6-311++G(2d,p) geometries as reported by DeBord et al.${ }^{57} ;{ }^{d}$ Experimental value from Ref.56; ${ }^{e}$ Experimental value from Ref. 7.

$\operatorname{CCSD}(\mathrm{T})$ calculations provide BDEs in very good agreement with threshold collision induced dissociation (TCID) experiments, that are reported to be $2.26 \pm 0.13 \mathrm{eV}$ and $1.55 \pm 0.13 \mathrm{eV}$ for $p$ $\mathrm{Me}-\mathrm{BnPy}^{+}$and $\mathrm{Me}, \mathrm{Me}-\mathrm{BhPy}{ }^{+}$, respectively. ${ }^{7,53}$ Note that our $\mathrm{CCSD}(\mathrm{T})$ results are also almost identical to recent calculations reported by Rahrt et al. at a very similar level of theory, ${ }^{7}$ DLPNO-CCSD/CBS//PBE0/D3BJ, reporting 2.26 and $1.52 \mathrm{eV}$ for $p-\mathrm{Me}-\mathrm{BnPy}^{+}$and $\mathrm{Me}, \mathrm{Me}-$ $\mathrm{BH}^{+}$, respectively, and by Schweikert and co-workers, ${ }^{57}$ reporting $2.267 \mathrm{eV}$ at $\mathrm{CCSD}(\mathrm{T}) / 6-$ $311++\mathrm{G}(\mathrm{d}, \mathrm{p}) / / \mathrm{B} 3 \mathrm{LYP} / 6-311++\mathrm{G}(2 \mathrm{~d}, \mathrm{p})$ level of theory for $p-\mathrm{Me}-\mathrm{BnPy}^{+}$.

Unfortunately, $\operatorname{CCSD}(\mathrm{T})$ method is computationally too expensive to be used in direct dynamics simulations. We have thus tested the capability of computationally cheaper methods in reproducing BDEs. We compare here values with and without ZPE. In fact, the former can be directly compared with experiments while the latter can be used to investigate how activation or threshold energies extracted from chemical dynamics compare with BDEs (trajectories are Newtonian and thus they do not have ZPE).

Concerning DFT results, the values obtained from M06-2X functional are globally more in agreement with experiments and $\operatorname{CCSD}(\mathrm{T})$ results than those from B3LYP. In the case of $p$ Me-BnPy ${ }^{+}, \mathrm{M}$ 06-2X BDEs are overestimated by about 0.1-0.2 eV, while B3LYP underestimate them by about $0.3-0.4 \mathrm{eV}$, and for Me,Me-BhPy ${ }^{+} \mathrm{M} 06-2 \mathrm{X}$ values are about $0.1-0.2 \mathrm{eV}$ off from experiments, while B3LYP provides much bigger difference (underestimation between 0.67 to $1.13 \mathrm{eV})$. Interestingly, M06-2X with a relatively small basis set like 6-31G(d) provides BDEs not far from the experiments. M06-2X/6-31G(d) method was thus used in DFT simulations. DFTB BDEs are underestimated by only $0.13 \mathrm{eV}$ and $0.10 \mathrm{eV}$ for $p-\mathrm{Me}_{\mathrm{BnPy}}{ }^{+}$and $\mathrm{Me}, \mathrm{Me}-$ $\mathrm{BhPy}^{+}$, respectively.

Between the different semi-empirical Hamiltonians used, PM6-D3 provides results that are more in agreement with experiments. In fact, $\mathrm{BDE}$ of $p-\mathrm{Me}-\mathrm{BnPy}^{+}$is underestimated by 0.17 $\mathrm{eV}$ and for $\mathrm{Me}, \mathrm{Me}-\mathrm{BhPy}^{+}$by $0.14 \mathrm{eV}$, which are close to the experimental uncertainty of 0.13 $\mathrm{eV}$. We have thus used PM6-D3 in chemical dynamics simulations as well.

Globally, few methods which can be used in chemical dynamics simulations provide BDE values which are inside the experimental uncertainty: M06-2X/6-311+G(d,p) for $p-\mathrm{Me}-\mathrm{BnPy}{ }^{+}$ and M06-2X/6-31G(d), PM6-D3 and DFTB for Me,Me-BhPy ${ }^{+}$. We have thus used M06-2X/631G(d), PM6-D3 and DFTB. Furthermore, for another ion of the BnPy family (the paramethoxy), we found relatively good agreement between values reported previously at CCSD(T) level of theory and calculated here, in particular when using M06-2X, DFTB and PM6-D3 (values are reported in Table S3).

Without taking into account the ZPE, the barrier increases by about 0.13 and $0.11 \mathrm{eV}$ for $p$-Me$\mathrm{BnPy}^{+}$and $\mathrm{Me}, \mathrm{Me}-\mathrm{BhPy}^{+}$, respectively. This should be considered in simulations, which are Newtonian and thus unimolecular dissociation will be slower than what is expected on the same potential energy surface including ZPE.

\subsection{Unimolecular Fragmentation Rate Constants}

Fragmentation dynamics were done at three levels of theory: M06-2X/6-31G(d), DFTB and PM6-D3. Both $p-\mathrm{Me}-\mathrm{BnPy}^{+}$and $\mathrm{Me}, \mathrm{Me}-\mathrm{BhPy}^{+}$provided only the expected fragmentation, i.e. 
the breaking of the $\mathrm{C}-\mathrm{N}$ bond, as a result of the simple unimolecular dissociation. It is thus possible to follow the decay of the precursor ion as a function of time for each value of energy (and corresponding temperature). As shown in Figure 1, we obtain exponential decays, such that it was possible to fit these curves to get unimolecular rate constants. Results of the fits are reported in Table 2.
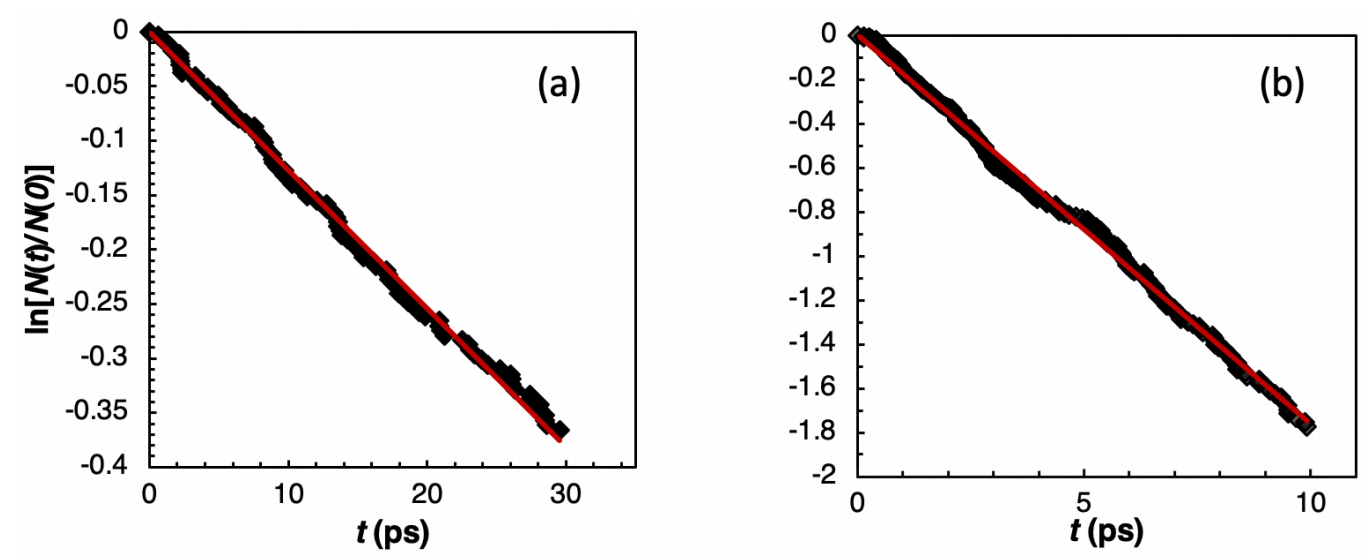

Figure 1. Plot of $\ln [N(t) / N(0)]$ vs time. for the simulations using DFTB for (a) $\mathrm{Me}-\mathrm{BnPy}^{+}$at $2266 \mathrm{~K}(15.18 \mathrm{eV})$ and (b) Me,Me-BhPy ${ }^{+}$at $2374 \mathrm{~K}(23.85 \mathrm{eV}) . N(t)$ is the number of surviving precursor ions at time $t$. The $\rho$ values for the fits are -0.9960 (panel a) and -0.9964 (panel b).

Table 2. Unimolecular rate constants (in $\mathrm{s}^{-1}$ ) obtained from unimolecular fragmentation during chemical dynamics simulations. Results are reported as a function of energy and temperature which are coupled by Eq. 2 .

\begin{tabular}{|c|c|c|c|c|c|}
\hline & $\begin{array}{l}\text { Energy } \\
(\mathrm{eV})\end{array}$ & $\begin{array}{l}\text { Temperature } \\
(\mathrm{K})\end{array}$ & M06-2X & DFTB & PM6-D3 \\
\hline \multicolumn{6}{|c|}{$p$-Me-BnPy ${ }^{+}$} \\
\hline & 10.84 & 1618.8 & - & - & $2.20 \pm 0.23 \cdot 10^{9}$ \\
\hline & 11.92 & 1780.6 & - & $9.00 \pm 0.29 \cdot 10^{8}$ & $6.30 \pm 0.14 \cdot 10^{9}$ \\
\hline & 13.00 & 1942.5 & - & $3.00 \pm 0.41 \cdot 10^{9}$ & $1.68 \pm 0.10 \cdot 10^{10}$ \\
\hline & 14.09 & 2104.4 & - & $5.90 \pm 0.30 \bullet 10^{9}$ & $3.18 \pm 0.08 \cdot 10^{10}$ \\
\hline & 15.18 & 2266.2 & $1.74 \pm 0.53 \cdot 10^{10}$ & $1.27 \pm 0.21 \cdot 10^{10}$ & $6.03 \pm 0.07 \cdot 10^{10}$ \\
\hline & 16.26 & 2428.1 & $3.16 \pm 0.47 \cdot 10^{10}$ & $2.68 \pm 0.16 \cdot 10^{10}$ & - \\
\hline & 17.34 & 2590.0 & $7.75 \pm 0.27 \cdot 10^{10}$ & $4.94 \pm 0.13 \cdot 10^{10}$ & $1.36 \pm 0.07 \cdot 10^{11}$ \\
\hline & 19.51 & 2913.8 & $2.00 \pm 0.21 \cdot 10^{10}$ & $1.25 \pm 0.12 \cdot 10^{11}$ & $2.50 \pm 0.09 \cdot 10^{11}$ \\
\hline & 21.68 & 3237.5 & $4.98 \pm 0.21 \cdot 10^{10}$ & $2.87 \pm 0.12 \cdot 10^{11}$ & - \\
\hline \multicolumn{6}{|c|}{$\mathrm{Me}, \mathrm{Me}-\mathrm{BhPy}^{+}$} \\
\hline & 10.84 & 1079.2 & - & - & $1.90 \pm 0.23 \cdot 10^{9}$ \\
\hline & 13.00 & 1295.0 & - & $3.00 \pm 0.77 \cdot 10^{8}$ & $1.20 \pm 0.10 \cdot 10^{10}$ \\
\hline & 15.18 & 1510.8 & - & $4.20 \pm 0.61 \cdot 10^{9}$ & $4.92 \pm 0.07 \cdot 10^{10}$ \\
\hline & 16.26 & 1618.8 & $6.17 \pm 0.31 \cdot 10^{10}$ & - & - \\
\hline & 17.34 & 1726.7 & $1.20 \pm 0.32 \cdot 10^{11}$ & $9.90 \pm 0.40 \bullet 10^{9}$ & $7.52 \pm 0.11 \cdot 10^{10}$ \\
\hline & 19.51 & 1942.5 & $3.29 \pm 0.22 \cdot 10^{11}$ & $3.27 \pm 0.20 \cdot 10^{10}$ & $1.79 \pm 0.15 \cdot 10^{11}$ \\
\hline & 21.68 & 2158.3 & $4.21 \pm 0.25 \cdot 10^{11}$ & $9.44 \pm 0.15 \cdot 10^{10}$ & $3.13 \pm 0.09 \cdot 10^{11}$ \\
\hline & 23.85 & 2374.2 & $5.86 \pm 0.22 \cdot 10^{11}$ & $1.76 \pm 0.12 \cdot 10^{11}$ & - \\
\hline
\end{tabular}

Trajectories were obtained by solving numerically the Newton's equations of motion and by on-the-fly calculations of energies and gradients using the three methods mentioned above. Resulting fragmentations thus reflect the motion on the classical potential energy surface (PES) 
and the associated BDEs are those reported without ZPE. The rate constants strongly depend on the method chosen because, as reported and discussed above, different methods provide different BDE values. This is particularly evident for lower energies (temperatures) which are closer to the barrier. For example, $p-\mathrm{Me}-\mathrm{BnPy}^{+}$at $13.0 \mathrm{eV}$ internal energy shows a rate constant of $3 \cdot 10^{9} \mathrm{~s}^{-1}$ at DFTB level of theory, while the fragmentation is one order of magnitude faster at PM6-D3 $\left(1.68 \cdot 10^{10} \mathrm{~s}^{-1}\right)$. This reflects the difference in BDE, which is lower at PM6-D3 level of theory than DFTB.

Due to the differences in BDEs calculated with each method, we sampled different energy values, that correspond to their fragmentation lifetimes, $(\tau=1 / k)$. In fact, it is computationally expensive to simulate very slow processes with accurate statistics. In other words, it is not possible to perform simulations at lower energies since the simulation time will grow exponentially. Energies close to the threshold would provide more accurate information on the barrier (but then the Arrhenius model could not be used): this is a limitation which one should consider in the following discussion. Since PM6-D3 shows faster reactivity with respect to DFTB we were able to simulate lower activation energies with this semi-empirical Hamiltonian, given that the two methods are computationally similar in terms of simulation time. M06-2X simulations are intrinsically computationally much slower and thus we could not simulate reactions with rate constants smaller than $10^{10} \mathrm{~s}^{-1}$. Note also that M06-2X BDEs are higher than those obtained by PM6-D3 and DFTB. Details on reactivity percentage as a function of method and system are reported in the supporting information in Tables S1 and S2.

\subsection{Activation, Threshold and Dissociation Energies}

Rate constants reported in section 3.2 are then used to estimate activation and threshold energies, using Arrhenius and RRK dependence of the rate constant. The equivalence with temperature is possible, as detailed in section 2.3, for classical systems with a large number of degrees of freedom and energies much higher than the threshold. Phenomenologically, it was also seen that peptide fragmentation follows an Arrhenius like behavior. ${ }^{11,12,47}$ Results for both $p$-Me-BnPy ${ }^{+}$and Me,Me-BhPy ${ }^{+}$reported in Figure 2 show that also in this case an Arrheniuslike behavior was observed. Thus, using Eq. 3 we obtain activation energies, which are reported in Table 3. The pre-exponential parameters are reported in the supporting information, Table S4.

Here we can evaluate the ability of a simple Arrhenius fit in estimating the BDE, by comparing $E_{a}$ with BDEs at the same level of theory and without ZPE, since the trajectories are Newtonian. For all methods and systems studied here, $E_{a}$ values are systematically lower than corresponding BDEs. For $p$-Me-BnPy ${ }^{+}, \mathrm{M} 06-2 \mathrm{X}$ and DFTB show the same deviation $(-0.36$ $\mathrm{eV}$ ) from the corresponding BDE (despite statistical sampling for M06-2X was much smaller than for DFTB), while PM6-D3 underestimates the value by $0.70 \mathrm{eV}$. On the other hand, for the larger system, Me,Me-BhPy ${ }^{+}, \mathrm{M}^{2} 6-2 \mathrm{X}$ simulations report the largest difference from the corresponding BDE $(-0.77 \mathrm{eV})$ whereas DFTB provides the fitted activation energy which is closer to its corresponding $\mathrm{BDE}(-0.08 \mathrm{eV})$. 

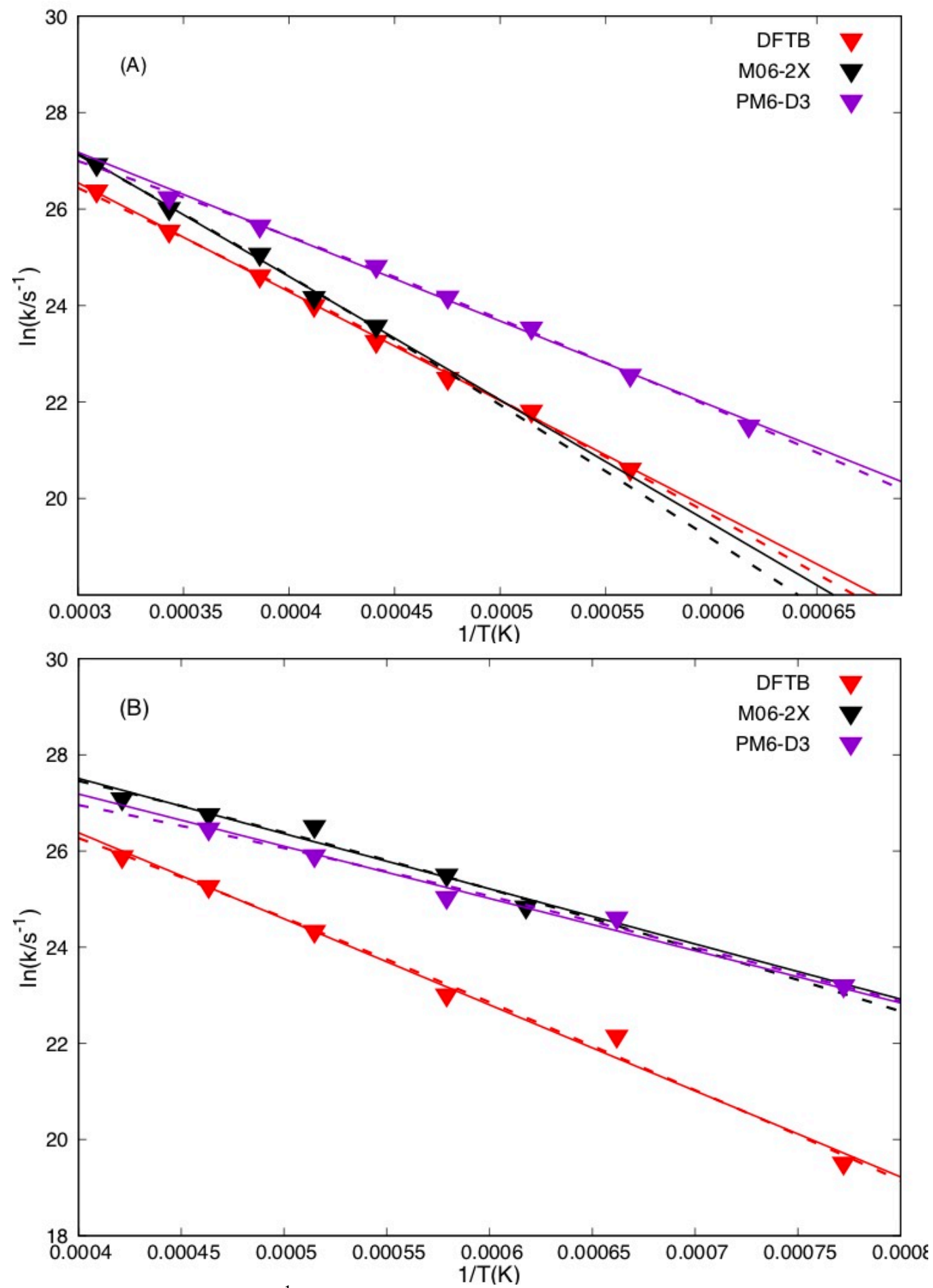

Figure 2. Rate constants $\left(\right.$ in s $\left.^{-1}\right)$ plotted against the inverse of temperature $(T$ in $\mathrm{K})$ for $p$-Me$\mathrm{BnPy}^{+}$and $\mathrm{Me}, \mathrm{Me}-\mathrm{BhPy}^{+}$simulations (panels $\mathrm{A}$ and $\mathrm{B}$, respectively). We report also the results of Arrhenius (continuous lines) and modified Arrhenius (dashed lines) fits (Eq. 3 and 4, respectively). 
Table 3. Activation, threshold and dissociation energies (in $\mathrm{eV}$ ) as obtained from Arrhenius and RRK fits. In brackets, we report the difference with respect to BDE value (without ZPE) as calculated for each system and method.

\begin{tabular}{|l|l|l|l|l|}
\hline & Method & $E_{a}{ }^{(1)}$ & $D_{0}{ }^{(2)}$ & $E_{0}{ }^{(3)}$ \\
\hline$p$-Me-BnPy & \multicolumn{4}{|l|}{} \\
\hline & M06-2X & $2.20 \pm 0.02$ & $2.78 \pm 0.09$ & $1.98 \pm 0.01$ \\
& & {$[-0.36]$} & {$[+0.22]$} & {$[-0.58]$} \\
\hline & DFTB & $1.95 \pm 0.03$ & $2.40 \pm 0.01$ & $1.74 \pm 0.03$ \\
& & {$[-0.36]$} & {$[+0.09]$} & {$[-0.57]$} \\
\hline & PM6-D3 & $1.51 \pm 0.05$ & $1.96 \pm 0.03$ & $1.37 \pm 0.03$ \\
& {$[-0.70]$} & {$[-0.25]$} & {$[-0.84]$} \\
\hline Me,Me-BhPy & \multicolumn{5}{|l}{} \\
\hline & M06-2X & $0.97 \pm 0.04$ & $1.40 \pm 0.11$ & $0.94 \pm 0.11$ \\
& & {$[-0.77]$} & {$[-0.34]$} & {$[-0.80]$} \\
\hline & DFTB & $1.52 \pm 0.03$ & $1.91 \pm 0.07$ & $1.41 \pm 0.06$ \\
& PM6-D3 & {$[-0.08]$} & {$[+0.31]$} & {$[-0.19]$} \\
\hline & $0.94 \pm 0.04$ & $1.25 \pm 0.04$ & $0.88 \pm 0.03$ \\
& {$[-0.59]$} & {$[-0.28]$} & {$[-0.65]$} \\
\hline MUE $^{(4)}$ & 0.48 & 0.25 & 0.60 \\
\hline
\end{tabular}

(1) Arrhenius fit as from Eq.3; ${ }^{(2)}$ modified Arrhenius fit with $m=-2.5$ as from Eq.4; ${ }^{(3)} R R K$ fit as from Eq.5; ${ }^{(4)}$ Mean Unsigned Error (in $\mathrm{eV}$ ) with respect to BDE values without $Z P E$.

As discussed in section 2.3, the Arrhenius activation energy is often lower than the known energy threshold, and this is quite general for unimolecular fragmentations characterized by loose TS. A generalized Arrhenius law (Eq. 4), which introduces a temperature dependence on the pre-exponential factor is often useful and is used here. In the present case, this introduces an additional parameter $m$, but as recently done for $\mathrm{CH}_{4}$ model fragmentation, ${ }^{13}$ the data can be more efficiently fitted by fixing $m$. After some tests, we found that $m=-2.5$ provides small uncertainties in the fitting parameters and thus we used this value. Results are reported in Tables 3 and S4. Note that the $m$ value is very close to what obtained for $\mathrm{CH}_{4}$ with the same approach (-2.5 and -2.4 in the present and $\mathrm{CH}_{4}$ study, respectively) and it is negative, reflecting the fact that $E_{a}<\mathrm{BDE}$. The modified-Arrhenius fit always results here in an improvement of the energy threshold estimation, except for the case of Me,Me-BhPy ${ }^{+}$with DFTB in which the simple Arrhenius expression provides $E_{a}$ very close to the corresponding BDE. Note that the differences between $E_{a}$ (Eq. 3) and $D_{0}$ (Eq. 4) reflects the $m k_{B} T$ difference, which is of the order of 0.4-0.5 eV depending on the temperatures sampled.

Microcanonical Newtonian simulations should follow an RRK behavior. In Figure 3 we show the energy dependence of the rate constants together with RRK fits. The threshold energies obtained by the RRK fit, reported in Table 3, are systematically underestimated with respect to the corresponding BDEs, following a behavior that is similar to what observed for $E_{a}$ values obtained from simple Arrhenius fits. 

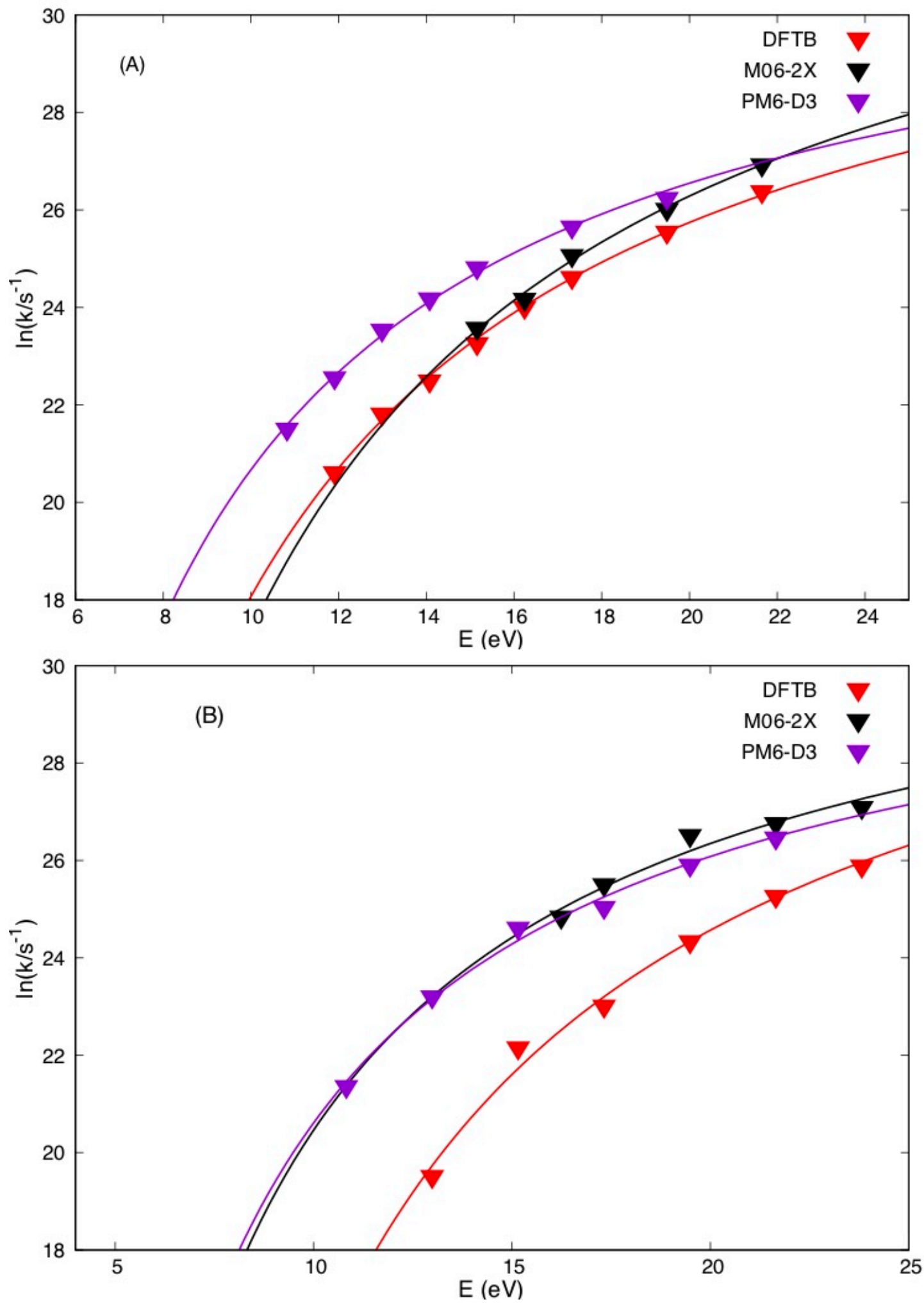

Figure 3. Rate constants as a function of internal energy and associated RRK fit as obtained from simulations of $p$-Me-BnPy ${ }^{+}$(panel A) and $\mathrm{Me}, \mathrm{Me}-\mathrm{BhPy}^{+}$(panel B).

Experimentally, rate constants were reported for the two systems, but only up to 6 and $4 \mathrm{eV}$ for $p$-Me-BnPy ${ }^{+}$and Me,Me-BhPy ${ }^{+}$, respectively, ${ }^{7,53}$ and thus a direct comparison cannot be done. However, since the values at the highest energies reported (about $4 \cdot 10^{9} \mathrm{~s}^{-1}$ and $10^{10} \mathrm{~s}^{-1}$ for $p$ $\mathrm{Me}-\mathrm{BnPy}^{+}$and $\mathrm{Me}, \mathrm{Me}-\mathrm{BhPy}^{+}$, respectively) are on the order of magnitude of what is found in simulations at higher energies, we can expect that simulations at low energies will largely 
underestimate the reaction rates. We should note that our extrapolations of rate constants at low energies can be poorly estimated for different reasons, like for example: (i) nuclear quantum effects are not taken into account in the present simulations; (ii) we cannot use Arrhenius-like expressions when $E \sim E_{a}$ (see section 2.3); (iii) we can use only the RRK expression which provides the worst description; (iv) simulations at low energies should be done, since finally the estimated $E_{o}$ depends on the energy range, but this is not possible even by using cheaper methods like PM6-D3 or DFTB. Surely this should be kept in consideration when using thermometer ions simulations to calibrate other simulations and when comparing with experiments. The limitation in simulating energies close to the threshold, can be overcome when further computer developments will allow to simulate longer time-scales.

One important point concerns the ability (or not) of Arrhenius and RRK plots to provide energy thresholds closer to BDE values. In general, the modified-Arrhenius equation provides $E_{a}$ values which are closer to the corresponding BDEs as compared to Arrhenius and RRK fitted values.

This finding is quantified by the mean unsigned error (MUE) calculated from the absolute difference between the activation or threshold energy, calculated from the fitting, and the BDE determined for each system and method (see Table 3). Clearly RRK fit provides the worst agreement, whereas the modified-Arrhenius provides values with the smallest MUE. Of course, the modified-Arrhenius expression has an extra parameter, improving the fit, but it also introduces a physical effect, namely the temperature dependence of the pre-exponential factor. In principle, it is possible to include energy dependence of pre-exponential factor and threshold energy in RRK theory, but the models available (also the simple one proposed by Song and Hase some years ago ${ }^{58}$ ) introduce too many parameters with multiple exponentials, making the fit totally unstable. We should note, in fact, that when the fragmentation passes through a looseTS, the energy threshold in RRK(M) theory has a temperature dependence, since the TS is defined from variational transition state theory (VTST). ${ }^{59}$ The simple fits used here clearly provide an average value for the energy region considered. This can be at the basis of the discrepancy between $E_{a}$ and $E_{o}$ and BDE. Note that in VTST the energy of the TS is always lower than the BDE, which is reflected in simple Arrhenius and RRK fit. Thus, to obtain $E_{o}$ values closer to BDE, without introducing any temperature or energy dependence in the rate constant expression, one should decrease the excess energy as much as possible in chemical dynamics simulations, which is computationally too slow to produce any meaningful result.

Quantitatively, while the MUE of modified-Arrhenius model is not small, we should notice that this changes as a function of the method employed. In particular, for $p$-Me- $\mathrm{BnPy}^{+}$system with DFTB the difference is only $0.09 \mathrm{eV}$. Again, DFTB has the smallest deviation from static BDE for $\mathrm{Me}, \mathrm{Me}-\mathrm{BhPy}^{+}$, in particular using a simple Arrhenius plot. To compare such barriers with experiments, we should note that simulations do not consider zero-point energy effects, which in the present case (as generally for dissociative processes with loose TS) has the net effect of lowering the energy barrier. We can now estimate from static calculations (see values reported in Table 1) this effect of the three methods and two systems and notably they are: $0.14,0.12$ and $0.20 \mathrm{eV}$ for $p-\mathrm{Me}-\mathrm{BnPy}^{+}$and $0.13,0.12$ and $0.15 \mathrm{eV}$ for Me,Me-BhPy ${ }^{+}$at M06-2X/6$31 \mathrm{G}(\mathrm{d})$, PM6-D3 and DFTB levels of theory, respectively. The resulting corrected barriers are reported in Table 4. We should note that DFTB simulations of $p-\mathrm{Me}-\mathrm{BnPy}^{+}$with modifiedArrhenius model provide a barrier value very similar to experiments. The same good performance is not obtained unfortunately for Me,Me-BhPy ${ }^{+}$, for which DFTB simulations with simple Arrhenius model perform slightly better. However, in general we should notice that DFTB is more in agreement with simulations. In fact, the MUA (considering the two systems 
and the three models) of DFTB is $0.33 \mathrm{eV}$, which is better than what obtained with M06-2X $(0.45 \mathrm{eV})$ and PM6-D3 $(0.71 \mathrm{eV})$. Concerning the models, also in this case the modifiedArrhenius model provides a better MUA (see Table 4). Note that this is a simple and crude estimation of the zero-point effect on the fragmentation barrier: more quantitative estimation should be done introducing some nuclear quantum effects directly in chemical dynamics simulations.

Table 4. Energy barriers obtained from the different models and simulation methods to which the corresponding zero-point energy effect was added as from static calculations (see Table 2). Experimental data are also reminded. All values are in $\mathrm{eV}$.

\begin{tabular}{|c|c|c|c|c|}
\hline & Method & $E_{a}^{+Z P E}$ & $D_{0}^{+Z P E}$ & $E_{0}{ }^{+Z P E}$ \\
\hline \multicolumn{5}{|c|}{$p$-Me-BnPy ${ }^{+}(\operatorname{Exp} .2 .26 \pm 0.13)^{a}$} \\
\hline & M06-2X & 2.06 & 2.64 & 1.84 \\
\hline & DFTB & 1.75 & 2.20 & 1.54 \\
\hline & PM6-D3 & 1.39 & 1.84 & 1.25 \\
\hline \multicolumn{5}{|c|}{ 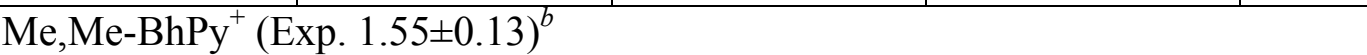 } \\
\hline & M06-2X & 0.84 & 1.27 & 0.81 \\
\hline & DFTB & 1.37 & 1.76 & 1.26 \\
\hline & PM6-D3 & 0.82 & 1.13 & 0.76 \\
\hline MUE $^{c}$ & & 0.53 & 0.29 & 0.66 \\
\hline
\end{tabular}

${ }^{a}$ Experimental value from Ref. $56 ;{ }^{b}$ Experimental value from Ref. $7 ;{ }^{c}$ Mean Unsigned Error with respect to experiments.

\subsection{Reaction products}

This section reports the fragmentation mechanisms of thermally excited $p$-Me-BnPy ${ }^{+}$and $\mathrm{Me}, \mathrm{Me}-\mathrm{BhPy}^{+}$during direct dynamics simulations using M06-2X/6-31G(d), DFTB, and PM6D3. Interestingly, for both ions the only bond dissociation observed is the $\mathrm{C}-\mathrm{N}$ bond, as shown in Scheme 1.

For both systems, M06-2X required substantially higher energies to achieve fragmentation. The lowest energies considered for $p$-Me-BnPy ${ }^{+}$and $\mathrm{Me}, \mathrm{Me}-\mathrm{BhPy}^{+}$using $\mathrm{M} 06-2 \mathrm{X}$ are 15.18 and $16.26 \mathrm{eV}$, respectively. In comparison, for DFTB the lowest energies are 11.92 and $13.0 \mathrm{eV}$ and $10.84 \mathrm{eV}$ for PM6-D3. DFT simulations are computationally much slower, thus shorter simulation times and less trajectories are simulated. Globally, the aim was to have enough reactive trajectories for each method to calculate kinetic parameters in sections 3.2 and 3.3. Here we now provide some important mechanistic aspects of the fragmentation.

Two competing fragmentation mechanisms have been suggested for benzylpyridinium ions. 55,60 The first mechanism involves a loose transition state with direct bond cleavage (DC) to give a benzylium cation and neutral pyridine molecule. ${ }^{55}$ Following the second mechanism, the system is supposed to undergo a rearrangement process (RP) to generate a tropylium ion via a tight transition state. ${ }^{55,60-62}$ De Pauw et al. found, during the experimental studies, that it is unlikely that the mechanism proceeds through RP instead it is solely due to DC. ${ }^{55}$ During the simulations, only the DC mechanism was observed for $p-\mathrm{Me}^{\mathrm{BnPy}}{ }^{+}$at all three levels of theory. As also reported by De Pauw, tropylium ion formation was not observed until C-N bond dissociated. They also noticed that harsher conditions and longer times in ion trap MS would lead to formation of tropylium whereas shorter times gave predominantly benzylium cation. Note that also Armentrout and co-workers provided experimental evidence that the tropylium cation is not formed. ${ }^{56}$ Our simulation study confirms all these findings. 
During thousands of simulations reported here for $p$-Me-BnPy ${ }^{+}$, the exclusive mechanism observed for product formation involved the formation of a benzylium cation. In small percentage of trajectories at 10.84 and $13.0 \mathrm{eV}$, using PM6-D3 method, the formation of tropylium ion was observed (3.9\% and $4.3 \%$ of reactive trajectories) but only after substantial time had passed following $\mathrm{C}-\mathrm{N}$ bond dissociation. In other terms, the mechanism involves breaking the $\mathrm{C}-\mathrm{N}$ bond to form the benzylium ion which later may rearrange to yield a tropylium ion (see Scheme 2 and a movie reported as supporting material, trop_full.mov, where unnecessary frames have been removed since benzylium to tropylium ion conversion takes a long time). For DFTB simulations tropylium ion formation was observed, in a similar fashion as in PM6-D3 simulations, at $21.68 \mathrm{eV}$ (1.7\% of reactive trajectories). This observation supports the previous findings that, if allowed, benzylium cation will form an equilibrium mixture favoring the thermodynamically more stable tropylium ion. ${ }^{55,63}$ In fact, the tropylium ion was found to be more stable than benzylium ion by $0.20 \mathrm{eV}$ with PM6-D3, $0.33 \mathrm{eV}$ with DFTB, and $0.32 \mathrm{eV}$ with M06-2X/6-31G(d). As reference, we should notice that both M06$2 \mathrm{X} / 6-311+\mathrm{G}(\mathrm{d}, \mathrm{p})$ and DLPNO-CCSD $(\mathrm{T}) / \mathrm{pVTZ}$ reports an energy difference of $0.25 \mathrm{eV}$, in agreement with experimental values for different $\mathrm{BnPy}^{+}$ions which are in the $0.2-0.4 \mathrm{eV}$ range. ${ }^{56}$ Tropylium ion formation was not observed in direct dynamics simulations with M06$2 \mathrm{X}$, likely because they were run for shorter time periods due to computationally expensive calculations.

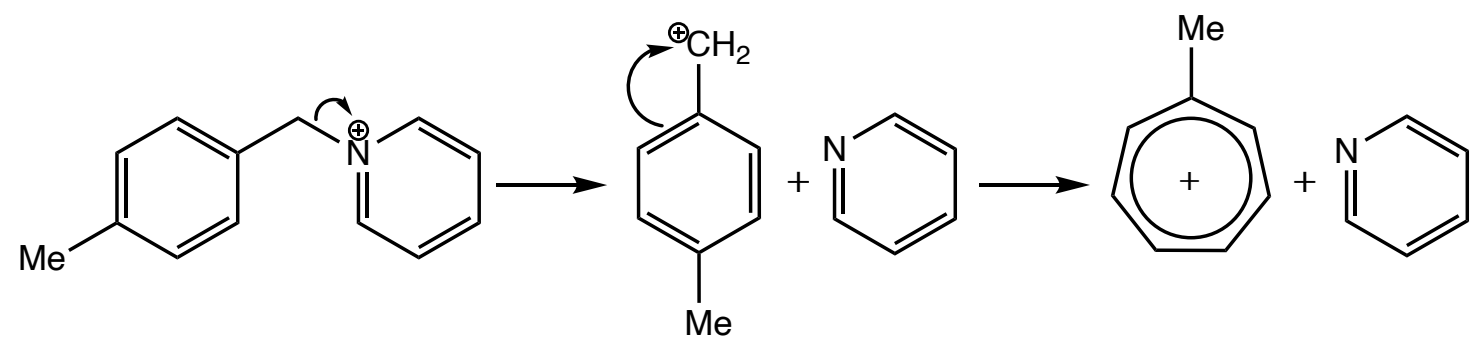

Scheme 2. Fragmentation mechanism for the formation of tropylium ion through benzylium isomer intermediate following $\mathrm{C}-\mathrm{N}$ bond dissociation in $\mathrm{p}-\mathrm{Me}-\mathrm{BnPy}{ }^{+}$.

In simulations of benzhydrylpyridinium ion we do not observe the formation of any other product ion, neither before nor after $\mathrm{C}-\mathrm{N}$ bond dissociation. This provides another advantage, in addition to the lower BDE, for using benzhydrylpyridinium ions as thermometer ions as compared to benzylpyrdinium ions (at least in experiments) since a competing dissociation pathway could compromise the reliability of the calibration procedure. ${ }^{60,61}$ In our simulations of Me,Me-BhPy ${ }^{+}$using M06-2X/6-31G(d), DFTB, and PM6-D3 there was no competing pathway observed as compared to dissociation of $\mathrm{C}-\mathrm{N}$ bond, forming a benzhydrylium ion and a neutral pyridine molecule, even though a wide range of energies $(10.84$ to $23.85 \mathrm{eV})$ were used to excite $\mathrm{Me}, \mathrm{Me}-\mathrm{BhPy}^{+}$in an attempt to model both softer and harsher experimental conditions.

\section{Conclusions}

In the present work, chemical dynamics simulations were employed to investigate the unimolecular fragmentation energy thresholds and mechanisms of two thermometer ions, $p$ $\mathrm{Me}-\mathrm{BnPy}^{+}$and Me,Me-BhPy ${ }^{+}$. The two systems have loose transition states and the threshold 
energies were used to determine the most efficient approach that can be used to estimate activation or threshold energies from chemical dynamics simulations.

Concerning the electronic structure theory, between the methods considered, M06-2X/631G(d), DFTB, and PM6-D3 provide the BDEs from static calculations that are most in agreement with experiments and highly-correlated calculations. They can be used, then, with some care, in subsequent fragmentation simulations.

From simulations, rate constants were directly extracted and used from Arrhenius and RRK kinetic theories to estimate activation and threshold energies. A comparison of these values with BDEs, calculated with the same theory, showed that the modified-Arrhenius results are more in agreement with corresponding BDEs. Note that when a reaction has a loose TS, the variational transition state theory is the appropriate theory to define it, and as a consequence its position along the reaction coordinate (and associated energy) depends on the activation energy. Simple Arrhenius and RRK theories do not consider it explicitly and thus the resulting activation and threshold energies are systematically underestimated with respect to the BDE. As briefly discussed in section 2.3 (and more developed in the Supporting Information) the two expressions are linked and the Arrhenius-like behavior can be applied only for excess energies that are much higher than the threshold energy. Also, low energies cannot be simulated due to the computational limitation in number of trajectories and simulation time-length. With the actual computational power and methods only high energies can be used in simulations leading to less accurate results and barriers which are systematically underestimated for fragmentations characterized by a loose transition state. Future developments in computational methods and/or faster methods which correctly describe fragmentation of ions will surely be useful to obtain more accurate information.

The modified-Arrhenius model introduces empirically a temperature dependence of the activation energy, which in many cases improves it with respect to corresponding theoretical BDE. The activation or threshold energies obtained from different electronic structure theories have different trends with respect to their corresponding BDEs (on average DFTB dynamical values are more similar to their corresponding static ones than M06-2X and PM6-D3): this is an interesting aspect which shows that other regions of the sampled potential energy surfaces also contribute to unimolecular fragmentation kinetics. Overall, we suggest using the modifiedArrhenius expression to evaluate activation energies from simulations in future studies.

Of course, the activation energies obtained from simulations should be, at a certain point, compared with experimental BDEs. The agreement depends on two aspects: (i) how does the electronic structure theory describe the potential energy surfaces and (ii) how do the trajectories and subsequent kinetic theories reproduce the BDE from activation or threshold energies. Considering the experimental uncertainty, one should look for values in the $2.13-2.39$ and 1.4 $-1.68 \mathrm{eV}$ range for $p-\mathrm{Me}-\mathrm{BnPy}^{+}$and $\mathrm{Me}, \mathrm{Me}-\mathrm{BhPy}^{+}$, respectively. Simulations do not consider zero-point energy effect, because they are Newtonian. However, taking into account in a qualitative way, the zero-point energy effect (i.e. simply modifying the barriers using the static ZPE effect on BDE), DFTB simulations provide results closer to experiments, in particular for the smaller system, $p$-Me-BnPy ${ }^{+}$. The worst agreement for $\mathrm{Me}, \mathrm{Me}-\mathrm{BhPy}^{+}$can have different origins, in particular in the electronic structure theory and in the sampling, which is smaller for a larger system. Overall, we suggest using DFTB with modified-Arrhenius model in further applications and developments. 
Clearly, the zero-point effect should be considered by adding nuclear quantum effects in simulations to have a fully dynamical approach. Recently we have done a first test on a simple model unimolecular fragmentation reaction including nuclear quantum effects via the quantum thermal bath method. ${ }^{13}$ Now, we are planning to apply the same approach to more complex systems with semi-empirical and/or DFTB electronic structure theories and the thermometer ions should be surely considered.

Thus, the present study opens the possibility of using chemical dynamics simulations to calibrate the energies of fragmentation processes. Notably, when performing simulations in this range of energy and time-length, it will be possible to compare the results with those of the thermometer ions and thus extrapolate the actual energy barrier. The known deviation of the energy barrier of the thermometer ions from experimental BDE should be considered. This will make possible to link the energy used in experimental collisionally activated dissociation (in ion traps the energy the ion gets is not univocally determined differently from other instruments, like triple-quadrupoles or the guided ion beam tandem mass spectrometer used to obtain the experimental BDEs compared here with our calculations) with the simulated ones.

Finally, simulations were able to show that the fragmentation of the thermometer ions occur only by direct cleavage of the $\mathrm{C}-\mathrm{N}$ bond. In the case of $p$-Me-BnPy ${ }^{+}$some tropylium ions were found, but only after the rearrangement of the primary fragmentation product, benzylium ion. These observations suggest that there is no competition between direct cleavage and a rearrangement process and thus no tight transition state is involved in fragmentation. Notably, in addition to its lower bond dissociation energy, $\mathrm{Me}, \mathrm{Me}-\mathrm{BhPy}^{+}$fragmentation never showed the formation of other product ions, suggesting that this system can be a better candidate to act as a thermometer ion than $p-\mathrm{Me}-\mathrm{BnPy}^{+}$.

\section{Acknowledgments}

The research at Texas Tech University (TTU) was supported by the Robert A. Welch Foundation under Grant No. D-0005. The simulations were performed with the computer clusters Chemdynm of the Hase Research Group and Quanah of the TTU High Performance Computing Center. We thank Dr. A.F. Perez-Mellor for help in VENUS/DFTB+ software. We also thank the anonymous reviewers which helped in improving the manuscript with their useful remarks. The authors would like to dedicate this work to the memory of Prof. W.L. Hase, who passed away too soon few weeks before the submission of this work.

\section{Supporting Information}

In the supporting material, we report: Tables with details on simulation conditions, bond dissociation energies of $p$-OMe- $\mathrm{BnPy}^{+}$at different levels of theory and pre-exponential parameters obtained from Arrhenius and RRK fits, derivation of equation 3 from equation 5 . We also report the video file trop_full.mov.

\section{References}

(1) Gabelica, V.; Pauw, E. De. Internal Energy and Fragmentation of Ions Produced in Electrospray Sources. Mass Spectrom. Rev. 2005, 24 (4), 566-587.

(2) Kenttämaa, H. I.; Cooks, R. G. Internal Energy Distributions Acquired through Collisional Activation at Low and High Energies. Int. J. Mass Spectrom. Ion Process. 1985, 64 (1), 79-83.

(3) Wysocki, V. H.; Kenttämaa, H. I.; Cooks, R. G. Internal Energy Distributions of Isolated Ions after Activation by Various Methods. Int. J. Mass Spectrom. Ion Process. 1987, 75 (2), 181-208.

(4) Cooks, R. G.; Ast, T.; Kralj, B.; Kramer, V.; Žigon, D. Internal Energy Distributions 
Deposited in Doubly and Singly Charged Tungsten Hexacarbonyl Ions Generated by Charge Stripping, Electron Impact, and Charge Exchange. J. Am. Soc. Mass Spectrom. 1990, 1 (1), 16-27.

(5) De Pauw, E.; Pelzer, G.; Marien, J.; Natalis, P. Internal Energy Distribution of Ions Emitted in Secondary Ion Mass Spectrometry. In Ion Formation from Organic Solids; Springer: Berlin, 1986; pp 103-108.

(6) Derwa, F.; de Pauw, E.; Natalis, P. New Basis for a Method for the Estimation Fo Seconary Ion Internal Energy Distribution in 'Soft' Ionization Techniques. Org. Mass Spectrom. 1991, 26 (2), 117-118.

(7) Rahrt, R.; Auth, T.; Demireva, M.; Armentrout, P. B.; Koszinowski, K. Benzhydrylpyridinium Ions: A New Class of Thermometer Ions for the Characterization of Electrospray-Ionization Mass Spectrometers. Anal. Chem. 2019, 91 (18), 11703-11711.

(8) Ofial, A. R. Benzhydrylium and Tritylium Ions: Complementary Probes for Examining Ambident Nucleophiles. Pure Appl. Chem. 2015, 87 (4), 341-351.

(9) Martin Somer, A.; Macaluso, V.; Barnes, G. L.; Yang, L.; Pratihar, S.; Song, K.; Hase, W. L.; Spezia, R. Role of Chemical Dynamics Simulations in Mass Spectrometry Studies of Collision-Induced Dissociation and Collisions of Biological Ions with Organic Surfaces. J. Am. Soc. Mass Spectrom. 2020, 31, 2-24.

(10) Pratihar, S.; Ma, X.; Homayoon, Z.; Barnes, G. L.; Hase, W. L. Direct Chemical Dynamics Simulations. J. Am. Chem. Soc. 2017, 139 (10), 3570-3590.

(11) Homayoon, Z.; Pratihar, S.; Dratz, E.; Snider, R.; Spezia, R.; Barnes, G. L.; Macaluso, V.; Martin-Somer, A.; Hase, W. L. Model Simulations of the Thermal Dissociation of the TIK $(\mathrm{H}+) 2$ Tripeptide: Mechanisms and Kinetic Parameters. J. Phys. Chem. A 2016, 120 (42), 8211-8227.

(12) Malik, A.; Lin, Y.-F.; Pratihar, S.; Angel, L.; Hase, W. Direct Dynamics Simulations of Fragmentation of a $\mathrm{Zn}(\mathrm{II})-2 \mathrm{Cys}-2 \mathrm{His}$ Oligopeptide. Comparison with Mass

Spectrometry Collision-Induced Dissociation. J. Phys. Chem. A 2019, 123 (32), 68686885.

(13) Spezia, R.; Dammak, H. On the Use of Quantum Thermal Bath in Unimolecular Fragmentation Simulation. J. Phys. Chem. A 2019, 123 (40), 8542-8551.

(14) Martín-Sómer, A.; Yáñez, M.; Gaigeot, M.-P.; Spezia, R. Unimolecular Fragmentation Induced By Low-Energy Collision: Statistically Or Dynamically Driven? J. Phys. Chem. A 2014, 118, 10882-10892.

(15) Molina, E. R.; Ortiz, D.; Salpin, J.-Y.; Spezia, R. Elucidating Collision Induced Dissociation Products and Reaction Mechanisms of Protonated Uracil by Coupling Chemical Dynamics Simulations with Tandem Mass Spectrometry Experiments. $J$. Mass Spectrom. 2015, 50 (12), 1340-1351.

(16) Troe, J. Theory of thermal unimolecular reactions at low pressures. I. Solutions of the master equation. J. Chem. Phys. 1977, 66, 4745-4757.

(17) Troe, J. Theory of thermal unimolecular reactions at low pressures. II. Strong collision rate constants. Applications. J. Chem. Phys. 1977, 66, 4758-4775.

(18) Troe, J. Specific rate constants k(E,J) for unimolecular bond fissions. J. Chem. Phys. 1983, 79, 6017.

(19) Troe, J. Simplified models for anharmonic numbers and densities of vibrational states. I. Application to $\mathrm{NO}_{2}$ and $\mathrm{H}_{3}{ }^{+}$. Chem. Phys. 1995, 190, 381-392.

(20) Rappe, A. K.; Casewit, C. J.; Colwell, K. S.; Goddard, W. A.; Skiff, W. M. UFF, a Full Periodic Table Force Field for Molecular Mechanics and Molecular Dynamics Simulations. J. Am. Chem. Soc. 1992, 114 (25), 10024-10035.

(21) Aradi, B.; Hourahine, B.; Frauenheim, T. DFTB+, a Sparse Matrix-Based 
mplementation of the DFTB Method. J. Phys. Chem. A 2007, 111 (26), 5678-5684.

Stewart, J. J. P. Optimization of Parameters for Semiempirical Methods VI: More Modifications to the NDDO Approximations and Re-Optimization of Parameters. $J$. Mol. Model. 2013, 19 (1), 1-32.

(23) Stewart, J. J. P. Optimization of Parameters for Semiempirical Methods V: Modification of NDDO Approximations and Application to 70 Elements. J. Mol. Model. 2007, 13 (12), 1173-1213.

(24) Grimme, S.; Antony, J.; Ehrlich, S.; Krieg, H. A Consistent and Accurate Ab Initio Parametrization of Density Functional Dispersion Correction (DFT-D) for the 94 Elements H-Pu. J. Chem. Phys. 2010, 132 (15), 154104.

(25) Dewar, M. J. S.; Zoebisch, E. G.; Healy, E. F.; Stewart, J. J. P. Development and Use of Quantum Mechanical Molecular Models. 76. AM1: A New General Purpose Quantum Mechanical Molecular Model. J. Am. Chem. Soc. 1985, 107 (13), 3902-3909.

(26) Rocha, G. B.; Freire, R. O.; Simas, A. M.; Stewart, J. J. P. RM1: A Reparameterization of AM1 for H, C, N, O, P, S, F, Cl, Br, and I. J. Comput. Chem. 2006, 27 (10), 11011111.

(27) Porezag, D.; Frauenheim, T.; Köhler, T.; Seifert, G.; Kaschner, R. Construction of Tight-Binding-like Potentials on the Basis of Density-Functional Theory: Application to Carbon. Phys. Rev. B 1995, 51 (19), 12947-12957.

(28) Seifert, G.; Porezag, D.; Frauenheim, T. Calculations of Molecules, Clusters, and Solids with a Simplified LCAO-DFT-LDA Scheme. Int. J. Quantum Chem. 1996, 58 (2), 185-192.

(29) Elstner, M.; Frauenheim, T.; Kaxiras, E.; Seifert, G.; Suhai, S. A Self-Consistent Charge Density-Functional Based Tight-Binding Scheme for Large Biomolecules. Phys. status solidi 2000, 217 (1), 357-376.

(30) Gaus, M.; Goez, A.; Elstner, M. Parametrization and Benchmark of DFTB3 for Organic Molecules. J. Chem. Theory Comput. 2013, 9 (1), 338-354.

(31) Becke, A. D. Density- functional Thermochemistry. III. The Role of Exact Exchange. J. Chem. Phys. 1993, 98 (7), 5648-5652.

(32) Stephens, P. J.; Devlin, F. J.; Chabalowski, C. F.; Frisch, M. J. Ab Initio Calculation of Vibrational Absorption and Circular Dichroism Spectra Using Density Functional Force Fields. J. Phys. Chem. 1994, 98 (45), 11623-11627.

(33) Zhao, Y.; Truhlar, D. G. The M06 Suite of Density Functionals for Main Group Thermochemistry, Thermochemical Kinetics, Noncovalent Interactions, Excited States, and Transition Elements: Two New Functionals and Systematic Testing of Four M06Class Functionals and 12 Other Function. Theor. Chem. Acc. 2008, 120 (1), 215-241.

(34) James J. P. Stewart. MOPAC2016. Stewart Comput. Chem. Color. Springs, CO, USA 2016, HTTP://OpenMOPAC.net.

(35) Valiev, M.; Bylaska, E. J.; Govind, N.; Kowalski, K.; Straatsma, T. P.; Van Dam, H. J. J.; Wang, D.; Nieplocha, J.; Apra, E.; Windus, T. L.; de Jong, W.A. NWChem: A Comprehensive and Scalable Open-Source Solution for Large Scale Molecular Simulations. Comput. Phys. Commun. 2010, 181 (9), 1477-1489.

(36) Hourahine, B.; Aradi, B.; Blum, V.; Bonafé, F.; Buccheri, A.; Camacho, C.; Cevallos, C.; Deshaye, M. Y.; Dumitrică, T.; Dominguez, A.; Ehlert, S.; Elstner, M.; van der H T.; Hermann, J.; Irle, S.; Kranz, J.J.; Köhler, C.; Kowalczyk, T.; Kubar, T.; Lee, I.S.; Lutsker, V.; Maurer, R.J.; Min, S.K.; Mitchell, I.; Negre, C.; Niehaus, T.A.; Niklasson, A.M.N.; Page, A.J.; Pecchia, A.; Penazzi, G.; Persson, M.P.; Rezac, J.; Sanchez, C.G.; Sternberg, M.; Stöhr, M.; Stuckenberg, F.; Tratchenko, A.; Yu, V.W.-Z.; Frauenheim, T. DFTB+, a Software Package for Efficient Approximate Density Functional Theory Based Atomistic Simulations. J. Chem. Phys. 2020, 152 (12), 124101. 
(37) Hase, W. L.; Buckowski, D. G. Monte Carlo Sampling of a Microcanonical Ensemble of Classical Harmonic Oscillators. Chem. Phys. Lett. 1980, 74 (2), 284-287.

(38) Schlier, C.; Seiter, A. Integration of Classical Trajectories: A Case Study. J. Phys. Chem. A 1998, 102, 9399-9404.

(39) Schlier, C.; Seiter, A. High-Order Symplectic Integration: An Assessment. Comput. Phys. Commun. 2000, 130 (1), 176-189.

(40) Verlet, L. Computer "Experiments" on Classical Fluids. I. Thermodynamical Properties of Lennard-Jones Molecules. Phys. Rev. 1967, 159, 98-103.

(41) Hase, W. L.; Duchovic, R. J.; Hu, X.; Komornicki, A.; Lim, K. F. .; Lu, D. H.; Peslherbe, G. H.; Swamy, K. N.; Vande Linde, S. R. .; Varandas, A.; Wang, H.; Wolf, R. J. VENUS. A General Chemical Dynamics Computer Program. Quantum Chem. Progr. Exch. Bull. 1996, 16 (4), 43.

(42) Hu, X.; Hase, W. L.; Pirraglia, T. Vectorization of the General Monte Carlo Classical Trajectory Program VENUS. J. Comput. Chem. 1991, 12 (8), 1014-1024.

(43) McQuarrie, D. A. Statistical Thermodynamics; Harper: New York, 1973.

(44) Yang, L.; Sun, R.; Hase, W. L. Use of Direct Dynamics Simulations to Determine Unimolecular Reaction Paths and Arrhenius Parameters for Large Molecules. J. Chem. Theory Comput. 2011, 7, 3478-3483.

(45) Lourderaj, U.; McAfee, J. L.; Hase, W. L. Potential Energy Surface and Unimolecular Dynamics of Stretched N-Butane. J. Chem. Phys. 2008, 129, 94701.

(46) Steinfeld, J. I.; Francisco, J. S.; Hase, W. L. Chemical Kinetics and Dynamics; Prentice-Hall: New York, 1999.

(47) Spezia, R.; Martin-Somer, A.; Macaluso, V.; Homayoon, Z.; Pratihar, S.; Hase, W. L. Unimolecular Dissociation of Peptides: Statistical vs. Non-Statistical Fragmentation Mechanisms and Time Scales. Faraday Discuss. 2016, 195 (0), 599-618.

(48) Kolakkandy, S.; Paul, A. K.; Pratihar, S.; Kohale, S. C.; Barnes, G. L.; Wang, H.; Hase, W. L. Energy and Temperature Dependent Dissociation of the Na+(Benzene) 1,2 Clusters. Importance of Anharmonicity. J. Chem. Phys. 2015, 142 (4), 44306.

(49) Johnston, H.; Birks, J. Activation Energies for the Dissociation of Diatomic Molecules Are Less than the Bond Dissociation Energies. Acc. Chem. Res. 1972, 5, 327-335.

(50) Marques, J. M. C.; Martínez-Núñez, E.; Fernández-Ramos, A.; Vázquez, S. A. Trajectory Dynamics Study of the Ar + CH4 Dissociation Reaction at High Temperatures: The Importance of Zero-Point-Energy Effects. J. Phys. Chem. A 2005, 109, 5415-5423.

(51) Levenberg, K. A Method for the Solution of Certain Non-Linear Problems in Least Squares. Q. Appl. Math. 1944, 2, 164-168.

(52) Marquardt, D. An Algorithm for Least-Squares Estimation of Nonlinear Parameters. SIAM J. Appl. Math. 1963, 11, 431-441.

(53) Williams, T.; Kelley, C. Gnuplot 5.2, 2007-2017. URL http://gnuplot.info. (Last accessed: 2020 October 6)

(54) Gatineau, D.; Memboeuf, A.; Milet, A.; Cole, R. B.; Dossmann, H.; Gimbert, Y.; Lesage, D. Experimental Bond Dissociation Energies of Benzylpyridinium Thermometer Ions Determined by Threshold-CID and RRKM Modeling. Int. J. Mass Spectrom. 2017, 417, 69-75.

(55) Morsa, D.; Gabelica, V.; Rosu, F.; Oomens, J.; De Pauw, E. Dissociation Pathways of Benzylpyridinium "Thermometer" Ions Depend on the Activation Regime: An IRMPD Spectroscopy Study. J. Phys. Chem. Lett. 2014, 5 (21), 3787-3791.

(56) Carpenter, J. E.; McNary, C. P.; Furin, A.; Sweeney, A. F.; Armentrout, P. B. How Hot Are Your Ions Really? A Threshold Collision-Induced Dissociation Study of Substituted Benzylpyridinium “Thermometer" Ions. J. Am. Soc. Mass Spectrom. 2017, 
$28(9), 1876-1888$.

(57) DeBord, J. D.; Verkhoturov, S. V.; Perez, L. M.; North, S. W.; Hall, M. B.; Schweikert E. A. Measuring the internal energies of species emitted from hypervelocity nanoprojectile impacts on surfaces using recalibrated benzylpyridinium probe ions. $J$. Chem. Phys. 2013, 138, 214301.

(58) Song, K.; Hase, W. L. Fitting classical microcanonical unimolecular rate constants to a modified RRK expression: Anharmonic and variational effects. J. Chem. Phys. 1999, 110, 6198-6207.

(59) Baer, T.; Hase, W. L. Unimolecular reaction dynamics: theory and experiments; Oxford University Press, New York, 1996.

(60) Zins, E.-L.; Pepe, C.; Rondeau, D.; Rochut, S.; Galland, N.; Tabet, J.-C. Theoretical and Experimental Study of Tropylium Formation from Substituted Benzylpyridinium Species. J. Mass Spectrom. 2009, 44 (1), 12-17.

(61) Zins, E.-L.; Rondeau, D.; Karoyan, P.; Fosse, C.; Rochut, S.; Pepe, C. Investigations of the Fragmentation Pathways of Benzylpyridinium Ions under ESI/MS Conditions. $J$. Mass Spectrom. 2009, 44 (12), 1668-1675.

(62) McLafferty, F. W.; Winkler, J. Gaseous Tropylium, Benzyl, Tolyl. and Norbornadienyl Cations. J. Am. Chem. Soc. 1974, 96 (16), 5182-5189.

(63) McLafferty, F. W.; Bockhoff, F. M. Formation of Benzyl and Tropylium Ions from Gaseous Toluene and Cycloheptatriene Cations. J. Am. Chem. Soc. 1979, 101 (7), 1783-1786. 


\section{For Table of Contents Use only}

Unimolecular Fragmentaion Properties of Thermometer Ions from Chemical Dynamics Simulations.

Abdul Malik, Riccardo Spezia and William L. Hase

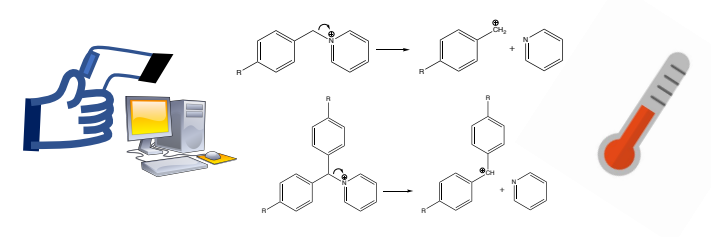

From chemical dynamics simulations, it is possible to extract molecular and energetic information of typical thermometer ions at different levels of theory for further use of them as calibration also for theoretical mass spectrometry studies. 